\title{
Blendas Biodegradáveis de Poli(3-Hidroxibutirato)/Poli(ع-Caprolactona): Obtenção e Estudo da Miscibilidade
}

\author{
Nilton Vogelsanger, Michele Cristina Formolo ${ }^{\mathrm{a}}$, Ana Paula Testa Pezzin ${ }^{\mathrm{a} *}$ \\ Andréa Lima dos Santos Schneider, Sandra Aparecida Furlan ${ }^{\mathrm{a}}$, \\ Heloísa Pinna Bernardo a, Sérgio Henrique Pezzin ${ }^{\text {b }}$, Alfredo Tibúrcio Nunes Pires ${ }^{c}$, \\ Eliana Aparecida de Rezende Duek ${ }^{\mathrm{d}}$ \\ ${ }^{\mathrm{a}}$ Universidade da Região de Joinville - UNIVILLE \\ Campus Universitário, C.P. 246, 89201-972 Joinville - SC, Brasil \\ ${ }^{\mathrm{b}}$ Universidade do Estado de Santa Catarina - UDESC, Joinville - SC, Brasil \\ ${ }^{\mathrm{c}}$ Universidade Federal de Santa Catarina - UFSC, Florianópolis - SC, Brasil \\ ${ }^{\mathrm{d}}$ Pontifícia Universidade Católica - PUC-SP, Sorocaba - SP, Brasil
}

Received: October 30, 2002; Revised: May 29, 2003

\begin{abstract}
Due to its biodegradability, poly(3-hydroxybutyrate) $\mathrm{P}(3-\mathrm{HB})$ has attracted much attention in the environmental sector. However, some characteristics of this polymer, such as high crystallinity, poor processability and high brittleness, have lead several research groups to study polymeric blends in order to modify $\mathrm{P}(3-\mathrm{HB})$ properties. Poly ( $\varepsilon$-caprolactone) (PCL) is a synthetic polyester which is completely degraded after about one year when buried in soil. In general, it acts as a polymeric plasticizer lowering the elastic modulus and enhancing the processability of the blend. Blends of two biodegradable polymers, $\mathrm{P}(3-\mathrm{HB})$ and PCL have been prepared by casting in different compositions. Miscibility, thermal behavior and morphology of these blends were studied using modulated differential scanning calorimetry (MDSC), scanning electron microscopy (SEM) and polarizing light microscopy (PLM). The two glass transition temperatures, detected by MDSC, suggest the immiscibility of the system. Phase separation was confirmed by PLM.
\end{abstract}

Keywords: blends, miscibility, $P(3-H B), P C L$

\section{Introdução}

Dentre os maiores problemas ambientais relacionados à utilização de materiais poliméricos, tais como o polipropileno, poliestireno, polietileno e poli(cloreto de vinila) pode ser destacado o tempo necessário para que ocorra degradação e a utilização de recursos não renováveis derivados do petróleo $^{1}$. Os poli(hidroxialcanoatos) (PHA), poliésteres naturais e biodegradáveis, podem sofrer degradação hidrolítica ou enzimática ${ }^{2-4} \mathrm{e}$ sob ação de microrganismos degradam completamente produzindo dióxido de carbono e água ${ }^{5,6}$. Devido a biodegradabilidade, o estudo de PHA tem despertado bastante interesse em aplicações na área ambiental. O poli(3-hidroxibutirato) $\mathrm{P}(3-\mathrm{HB})$ é produzido através de fermentação bacteriana, com predominância de utilização da bactéria Raltonia eutroph $a^{7}$. A biossíntese deste polímero permite um processo cíclico sustentável através de fontes renováveis, substituindo tecnologias de ponta ligadas à produção e ao uso de materiais poliméricos sintéti$\cos ^{8}$. Entretanto, a baixa processabilidade, o elevado grau de cristalinidade e fragilidade do $\mathrm{P}(3-\mathrm{HB})$ limitam suas aplicações. A fim de se obter materiais com melhores características, misturas físicas de $\mathrm{P}(3-\mathrm{HB})$ com outros polímeros (blendas) também biodegradáveis podem ser preparadas. O estudo dos sistemas $\mathrm{P}(3-\mathrm{HB}) /$ poli(propiolactona), $\mathrm{P}(3-\mathrm{HB}) /$ poli(adipato de etileno) e $\mathrm{P}(3-\mathrm{HB}) /$ poli(3-hidroxibutirato-co-3-hidroxivalerato) (PHBV) mostrou que a cinética de degradação das blendas é mais acelerada quando comparada com os polímeros puros, sendo atribuído ao processo de separação de fases dos componentes nas blendas ${ }^{9}$. Trabalhos anteriores mostraram que é possível

*e-mail: paulapezzin@univille.edu.br

Trabalho apresentado no 1ํㅡㄹ Congresso da Sociedade Brasileira de Pesquisa em Materiais, Rio de Janeiro, julho de 2002. 
controlar a taxa de degradação hidrolítica dos componentes das blendas, por exemplo, acelerando a degradação de poli(L-ácido láctico) (PLLA) através da adição poli( $p$-dioxanona) (PPD) ${ }^{10,11}$. Poli( $\varepsilon$-caprolactona) (PCL) pode agir como plastificante polimérico, diminuindo o módulo de rigidez e melhorando a processabilidade da blenda ${ }^{12}$. Neste trabalho foram preparadas blendas de $\mathrm{P}(3-\mathrm{HB}) / \mathrm{PCL}$ e avaliadas a miscibilidade, buscando obter materiais flexíveis e biodegradáveis, para aplicações na área agrícola.

\section{Parte Experimental}

\section{Materiais}

$\mathrm{O} \mathrm{P}(3-\mathrm{HB})$ utilizado foi gentilmente fornecido pela $\mathrm{PHB}$ Industrial (lote FE-67 ESF) identificado por espectroscopia no infravermelho (FTIR) da Perkin Elmer Spectrum One, no intervalo de 4000 a $650 \mathrm{~cm}^{-1}$ pela técnica de reflectância difusa e difratometria de raios-X (DRX) da SHIMATZU modelo XRD 6000, variando o ângulo $2 \theta$ de 15 a $35^{\circ}$ e utilizando fonte de $\mathrm{CuK} \alpha$.

A massa molar viscosimétrica foi determinada em clorofórmio a $30{ }^{\circ} \mathrm{C}$ a partir da equação de Mark-Houwink (Eq. 1):

$$
[\eta]=\mathrm{k} \mathrm{M}^{\alpha}
$$

onde [ $\eta$ é a viscosidade intrínseca e k e $\alpha$ são as constantes para o sistema polímero-solvente. Utilizando os valores de $\mathrm{k}=1,18 \times 10^{-4}$ e $\alpha=0,78^{13}$, foi obtido a massa molar média do P(3-HB) igual a $342.000 \mathrm{~g} / \mathrm{mol}$. A PCL da Sigma-Aldrich com $M_{w}$ igual a $60.000 \mathrm{~g} / \mathrm{mol}$, segundo o fabricante. As fórmulas estruturais das unidades monoméricas dos polímeros são apresentadas nas Fig. 1. O clorofórmio, utilizado como solvente foi fornecido pela Synth.

\section{Preparação das Blendas}

Inicialmente, foram preparadas soluções de $\mathrm{P}(3-\mathrm{HB}) \mathrm{a}$ $1 \% \mathrm{~m} / \mathrm{v}$ sob aquecimento e PCL na mesma concentração a temperatura ambiente, utilizando clorofórmio como solvente



(a)

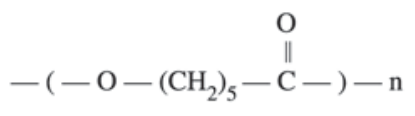

(b)

Figura 1. Estruturas químicas: a) poli(3-hidroxibutirato); b) da poli( $\varepsilon$-caprolactona). comum. Em seguida foram misturadas diferentes proporções de cada uma das soluções e mantidas sob agitação durante $2 \mathrm{~h}$, de maneira a obter as composições de 0/100, 20/80, 50/50, 80/20 e 100/0 m/m de P(3-HB)/PCL. Os filmes foram obtidos por evaporação do solvente em atmosfera saturada de clorofórmio por $48 \mathrm{~h}$, mantidos sob vácuo durante $24 \mathrm{~h}$ e armazenadas em dessecador.

\section{Caracterização das Blendas}

Calorimetria Diferencial de Varredura Modulada (MDSC)

As medidas no MDSC foram feitas no equipamento TA Instruments, modelo 2920. As amostras foram seladas em recipientes de alumínio, aquecidas de 25 a $200{ }^{\circ} \mathrm{C}$ a $10^{\circ} \mathrm{C} / \mathrm{min}$ e mantidas nesta temperatura por $5 \mathrm{~min}$. Em seguida, as amostras foram resfriadas a $-100{ }^{\circ} \mathrm{C}$ numa taxa de $30{ }^{\circ} \mathrm{C} / \mathrm{min}$. Após $5 \mathrm{~min}$ a $-100{ }^{\circ} \mathrm{C}$ as amostras foram submetidas a um segundo aquecimento até $200{ }^{\circ} \mathrm{C}$, com taxa de $5{ }^{\circ} \mathrm{C} / \mathrm{min}$. A amplitude e o período de oscilação utilizados foram $0,5^{\circ} \mathrm{C}$ e $60 \mathrm{~s}$, respectivamente.

Microscopia Eletrônica de Varredura (SEM)

Os fragmentos da superfície superior e da superfície da fratura criogênica dos filmes foram fixados em suporte metálico e recobertos com ouro, utilizando-se um metalizador de amostras Sputer Coater BAL-TEC SCD 050 para obtenção das micrografias. Foi utilizado um microscópio eletrônico de varredura JEOL JXA 860A, utilizando tensão de $20 \mathrm{kV}$.

\section{Microscopia Óptica com luz polarizada (PLM)}

Filmes com cerca de $5 \mu \mathrm{m}$ de espessura foram prensados a partir do fundido a $190{ }^{\circ} \mathrm{C}$ por 1 min e colocados entre duas lamínulas para observação no microscópio óptico. Antes de cristalizadas, as amostras foram primeiramente aquecidas a $190{ }^{\circ} \mathrm{C}$ por $1 \mathrm{~min}$ em um sistema de aquecimento da Mettler e em seguida resfriadas até a temperatura de cristalização de $83{ }^{\circ} \mathrm{C}$ e temperatura ambiente para o $\mathrm{P}(3-\mathrm{HB})$ e PCL, respectivamente. O microscópio óptico com luz polarizada Olympus BX-50 equipado com controle de aquecimento foi utilizado para avaliar as morfologias das blendas a diferentes composições.

\section{Resultados e Discussão}

O perfil de difração de raios-X (DRX) para o $\mathrm{P}(3-\mathrm{HB})$ é mostrado na Fig. 2. Como pode-se observar, o P(3-HB) apresenta picos de difração em $2 \theta$ igual a 13,5; 17; 22 e 25,5 , sendo similar ao padrão mostrado por Ikejima e Inoue, $2000{ }^{14}$. O grau de cristalinidade $\left(\alpha_{c}\right)$ determinado para o polímero foi igual a $48,5 \%$. O espectro de FTIR mostrado na Fig. 3 demonstra que o polímero doado pela PHB Industrial apresenta bandas características do $\mathrm{P}(3-\mathrm{HB})$, tais como as das deformações axiais da carbonila $(\mathrm{C}=\mathrm{O})$ a $1737 \mathrm{~cm}^{-1}$, 
de O-H a $3438 \mathrm{~cm}^{-1}$ e de C-C a $978 \mathrm{~cm}^{-1}$, além da banda de deformação angular simétrica no plano dos grupos $\mathrm{CH}_{3} \mathrm{em}$ $1380 \mathrm{~cm}^{-1}$ e a banda típica da conformação helicoidal das cadeias a $1227 \mathrm{~cm}^{-1}$. As bandas a $1134 \mathrm{e} 1187 \mathrm{~cm}^{-1}$ são atribuídas, respectivamente, aos estiramentos simétrico e assimétrico do grupo C-O-C ${ }^{15}$.

Calorimetria Diferencial de Varredura Modulada (MDSC)

O primeiro aquecimento foi realizado para apagar a história térmica das blendas e poder compará-las em condições térmicas similares. A Fig. 4 mostra as curvas de MDSC para o resfriamento, as quais foram utilizadas para a determinação das temperaturas $\left(\mathrm{T}_{c}\right)$ e entalpias de cristalização $\left(\Delta \mathrm{H}_{\mathrm{c}}\right)$. Durante o resfriamento houve um decréscimo dos

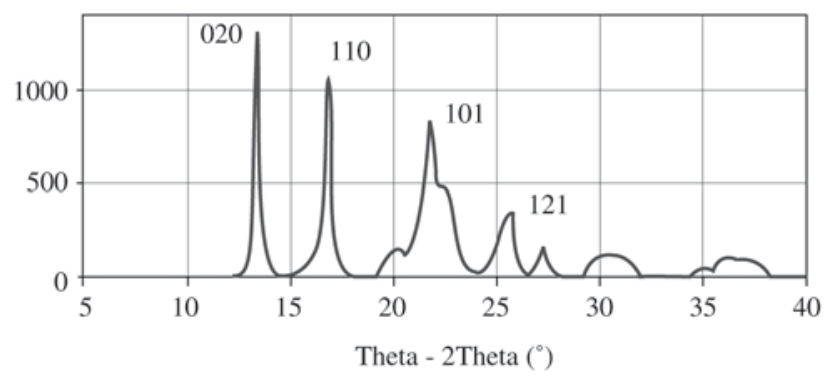

Figura 2. DRX do $\mathrm{P}(3-\mathrm{HB})$. valores de $\mathrm{T}_{c}$ do $\mathrm{P}(3-\mathrm{HB})$ com o aumento da concentração de PCL nas blendas, sendo que para concentrações de PCL acima de $50 \%$ a $\mathrm{T}_{\mathrm{c}}$ do $\mathrm{P}(3-\mathrm{HB})$ não foi detectada pela técnica utilizada. Por outro lado os valores de $T_{c}$ do PCL e as entalpias de cristalização $\left(\Delta \mathrm{H}_{c}\right)$ de ambos os polímeros não sofreram alteração em função da composição (Fig. 5a e 5b, respectivamente). Na Fig. 6 são apresentadas as curvas de MDSC para o segundo aquecimento referente aos componentes puros e blendas em diferentes composições, cujos valores obtidos para $\mathrm{T}_{\mathrm{g}}$ foram $2 \mathrm{e}-67{ }^{\circ} \mathrm{C}$ para o $\mathrm{P}(3-\mathrm{HB}) \mathrm{e}$ PCL, respectivamente, concordando com os valores da literatura, ${ }^{9}$. A Fig. 7 mostra que as temperaturas de transição vítrea da PCL e P(3-HB) em função do aumento do conteúdo de PCL nas blendas permaneceram na mesma ordem de grandeza para todas composições estudadas, sugerindo que os polímeros são imiscíveis na fase amorfa. Esta observação concorda com os resultados de Kumagai e Doi para o mesmo sistema, sendo as blendas preparadas por moldagem por compressão. Neste estudo, a $\mathrm{T}_{\mathrm{g}}$ do $\mathrm{P}(3-\mathrm{HB})$ permaneceu em torno de $1{ }^{\circ} \mathrm{C}$, porém os autores não conseguiram observar a $\mathrm{T}_{\mathrm{g}}$ da PCL na blenda, devido à alta cristalinidade deste polímero?.

Nas curvas de MDSC (Fig. 6) foram observados dois picos endotérmicos a 168 e $55^{\circ} \mathrm{C}$, devido à fusão das fases cristalinas do $\mathrm{P}(3-\mathrm{HB})$ e da PCL, respectivamente. Quental e col $^{15}$ obtiveram dois valores de $\mathrm{T}_{\mathrm{f}}$ do $\mathrm{P}(3-\mathrm{HB})$ a $167 \mathrm{e}$ $147^{\circ} \mathrm{C}$, sendo que o segundo pico não foi observado em

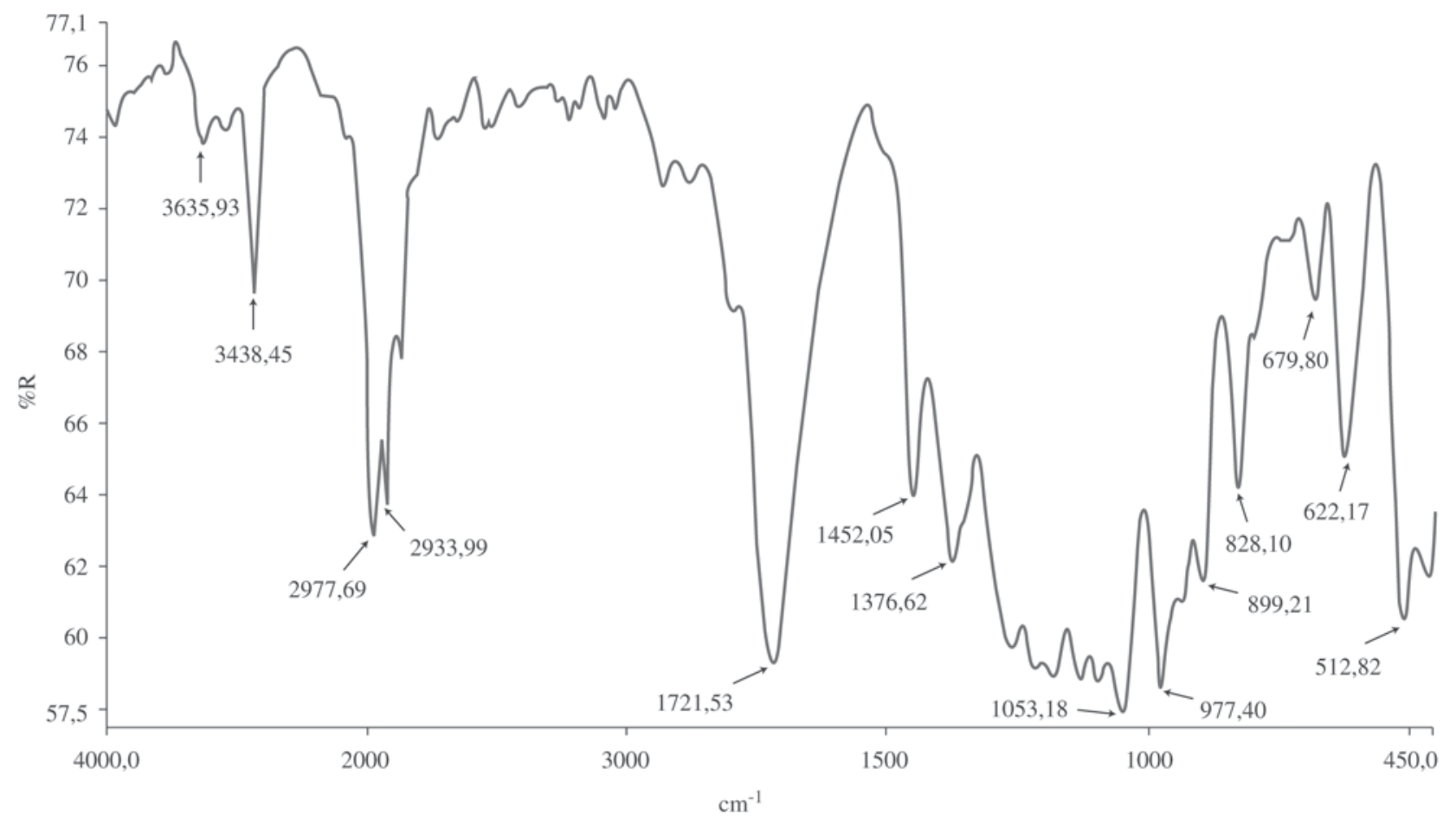

Figura 3. FTIR do $\mathrm{P}(3-\mathrm{HB})$. 


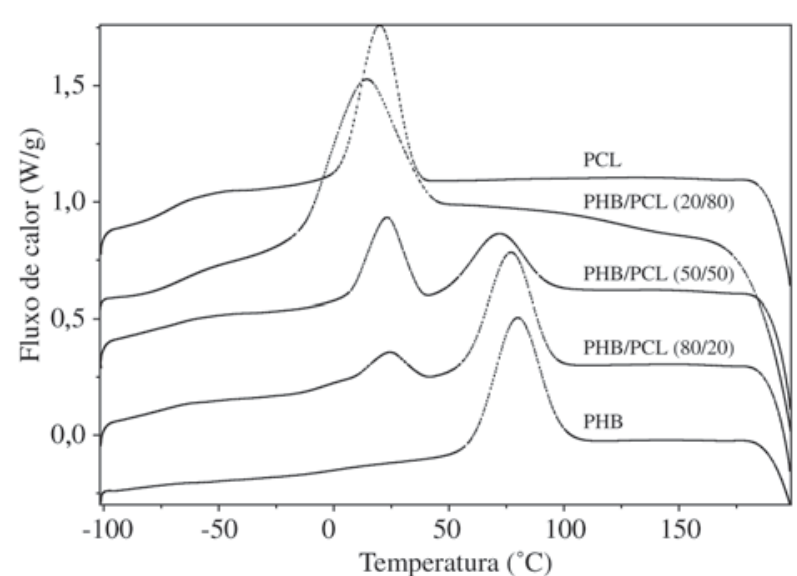

Figura 4. Curvas de fluxo de calor total, obtidas por MDSC para as blendas $\mathrm{P}(3-\mathrm{HB}) / \mathrm{PCL}$ no resfriamento.
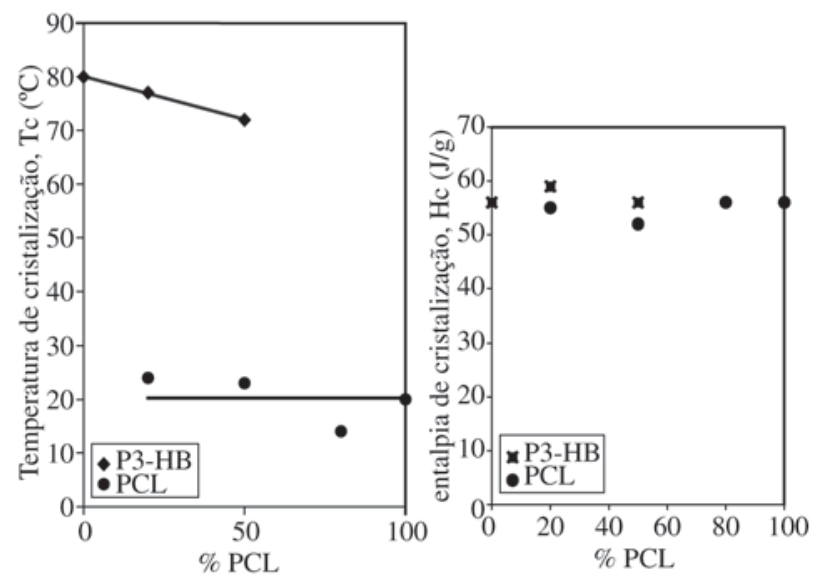

Figura 5. a) Temperaturas de cristalização do P(3-HB) e da PCL em função da \% de PCL na blenda; b) Entalpias de cristalização do P(3-HB) e da PCL em função da \% de PCL na blenda.

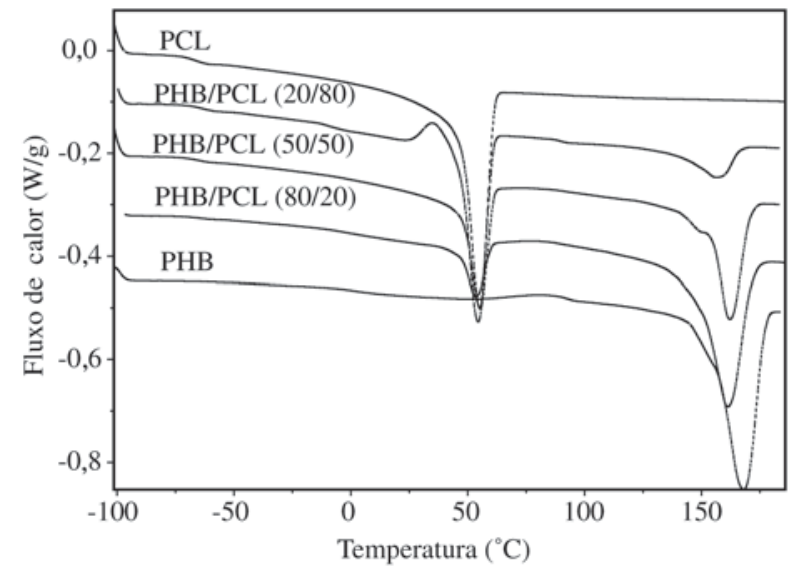

Figura 6. Curvas de fluxo de calor total, obtidas por MDSC para as blendas $\mathrm{P}(3-\mathrm{HB}) / \mathrm{PCL}$ no segundo aquecimento. nosso experimento. O gráfico da Fig. 8a mostra para todas as composições que a $\mathrm{T}_{f}$ da PCL permaneceu constante e a $\mathrm{T}_{f}$ do $\mathrm{P}(3-\mathrm{HB})$ decresceu com o aumento da percentagem de PCL na blenda. A diminuição da $\mathrm{T}_{f}$ do $\mathrm{P}(3-\mathrm{HB})$ sugere que a composição da blenda influencia na cristalização do $\mathrm{P}(3-\mathrm{HB})$, podendo ser um reflexo da morfologia e das propriedades térmicas dos polímeros.

Como observado na temperatura de fusão, os valores de $\Delta \mathrm{H}_{f}$ do $\mathrm{P}(3-\mathrm{HB})$ permaneceram inalterados para as diferentes composições das blendas, enquanto para o PCL foi verificado um decréscimo do $\Delta \mathrm{H}_{f}$ em função do aumento da composição do PCL na blenda (Fig. 8b). A partir dos dados de $\Delta \mathrm{H}_{f}$ e entalpia de fusão para o polímero $100 \%$ cristalino,

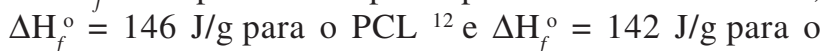
$\mathrm{P}(3-\mathrm{HB}){ }^{16}$, o grau de cristalinidade dos componentes na blenda foi calculado $\left(\alpha_{c} \%\right)$, seguindo a Eq. 2:

$$
\alpha_{\mathrm{c}}=\frac{\Delta H_{f}}{\Delta H_{f}^{o}} \times 100
$$

A Fig. 9 apresenta a variação do grau de cristalinidade do P(3-HB) e do PCL em função do aumento percentagem de PCL na blenda. Para os polímeros puros foi obtido $\alpha_{c}$ igual a $51 \%$ para o $\mathrm{P}(3-\mathrm{HB})$ e $45 \%$ para a PCL. Observa-se que os valores de cristalinidade obtidos por MDSC e DRX foram muito próximos, $51 \%$ e $48,5 \%$, respectivamente. O grau de cristalinidade da PCL tende a diminuir com o aumento do conteúdo de PCL, enquanto o grau de cristalinidade do $\mathrm{P}(3-\mathrm{HB})$ permanece constante.



Figura 7. Temperaturas de transição vítrea do P(3-HB) e da PCL em função da \% de PCL na blenda. 

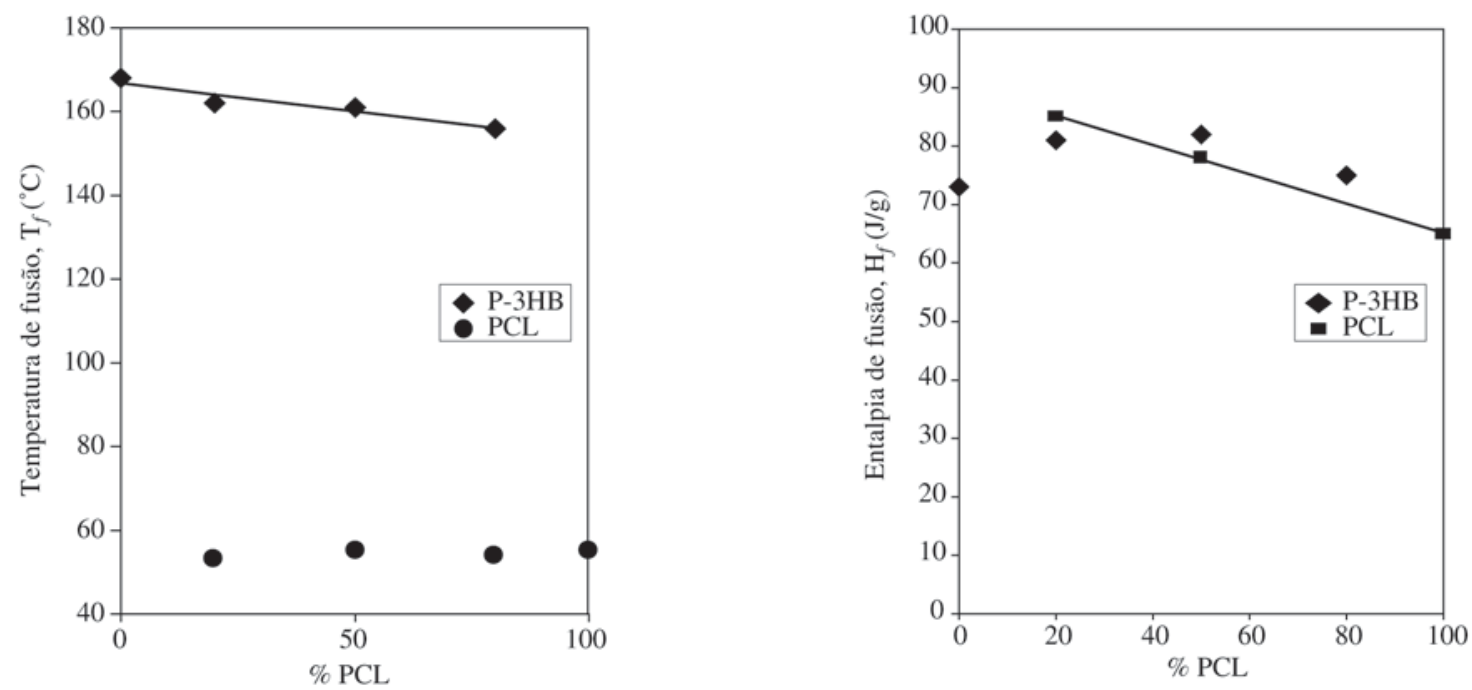

Figura 8. a) Temperaturas de fusão do P(3-HB) e da PCL em função da \% de PCL na blenda; b) Entalpias de fusão do P(3-HB) e da PCL em função da \% de PCL na blenda.

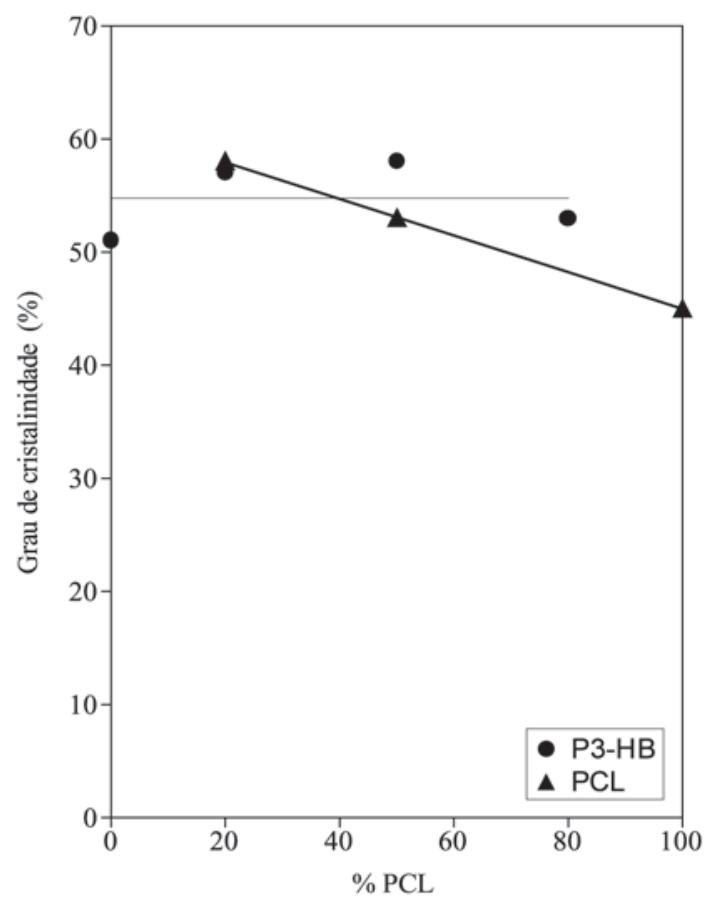

Figura 9. Grau de cristalinidade em função da \% de PCL.

\section{Microscopia Eletrônica de Varredura (SEM)}

As micrografias de SEM da superfície superior dos polímeros puros mostram uma superfície rugosa e irregular para o P(3-HB) e para a PCL observa-se uma superfície lisa, descontínua, com cristais limitados pelos arranjos cris- talinos vizinhos, formando polígonos (Figs. 10a, 10c). A morfologia variou bastante em função da composição das blendas, sendo que a composição 50/50, constituída por microesferas com cerca de $8 \mathrm{~mm}$, foi a que apresentou a morfologia mais diferenciada (Fig. 10b).

Microscopia Óptica com luz polarizada (PLM)

A Fig. 11a apresenta a micrografia obtida por PLM com luz polarizada para o $\mathrm{P}(3-\mathrm{HB})$, mostrando um típico esferulito deste polímero, no qual se observam anéis concêntricos regulares. A porção externa apresenta pequenos cristais inseridos na massa fundida, que não fundiram a $190{ }^{\circ} \mathrm{C}$, atribuídos provavelmente à impureza do polímero. A micrografia da PCL (Fig. 11f) apresenta os esferulitos da PCL, cristalizados a temperatura ambiente. Estes esferulitos são bem pequenos, pois uma enorme quantidade de núcleos foram formados com baixa velocidade de cristalização, devido ao fato da isoterma de cristalização ter sido realizada acima da temperatura de cristalização da PCL. A Fig. 11c corresponde à blenda $\mathrm{P}(3-\mathrm{HB}) / \mathrm{PCL} 50 / 50$, onde foi realizada uma isoterma a $83{ }^{\circ} \mathrm{C}$. A micrografia apresenta, nesta temperatura, o $\mathrm{P}(3-\mathrm{HB})$ já cristalizado formando esferulitos enquanto o PCL está ainda fundido, uma vez que sua temperatura de cristalização é bem abaixo da isoterma realizada. Já na Fig. 11d, correspondente a blenda P(3-HB)/PCL 50/50, observa-se uma total imiscibilidade entre os domínios de P(3-HB) e PCL, confirmando os dados observados por SEM. Nesta micrografia, nota-se a presença de esferulitos do $\mathrm{P}(3-\mathrm{HB})$ (anel na parte superior à esquerda) $\mathrm{e}$ de PCL (restante), pois foi realizada uma isoterma a $83^{\circ} \mathrm{C}$ para cristalização do $\mathrm{P}(3-\mathrm{HB})$, seguida de resfriamento a 


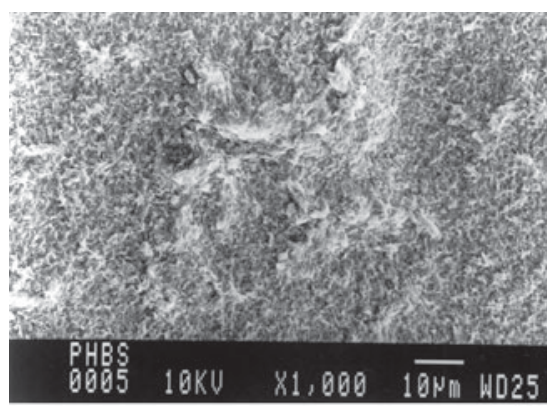

(a)

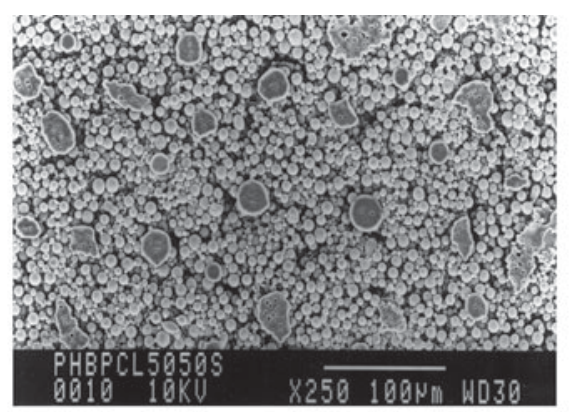

(b)

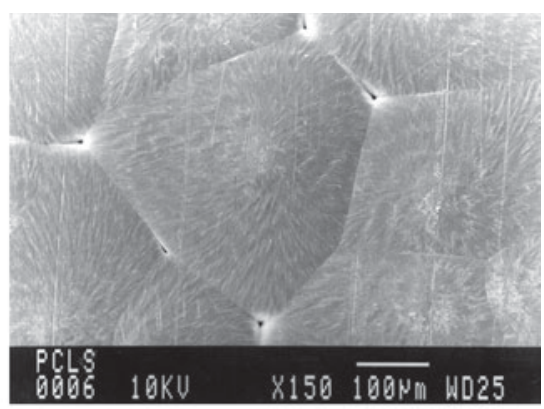

(c)

Figura 10. Micrografias de SEM da superfície superior das blendas de $\mathrm{P}(3-\mathrm{HB}) / \mathrm{PCL}$ e dos homopolímeros: a) P(3-HB); b) P(3-HB)/PCL $(50 / 50)$; c) PCL.
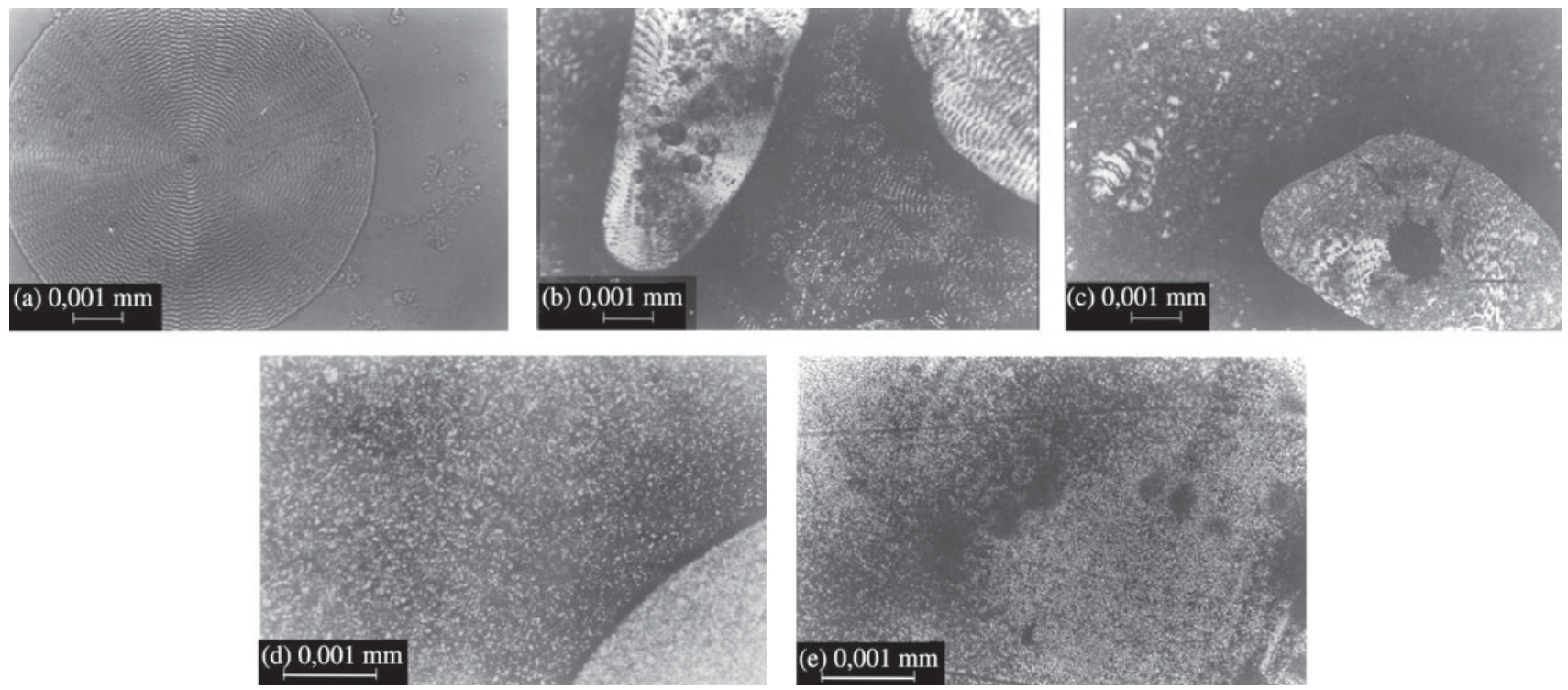

Figura 11. Micrografias de PLM com luz polarizada das blendas de $\mathrm{P}(3-\mathrm{HB}) / \mathrm{PCL}$ e dos homopolímeros: a) $\mathrm{P}(3-\mathrm{HB})$; b) $\mathrm{P}(3-\mathrm{HB}) / \mathrm{PCL}$ (80/20); c) P(3-HB)/PCL (50/50); d) P(3-HB)/PCL (20/80); e) PCL.

temperatura ambiente e outra isoterma a $25^{\circ} \mathrm{C}$ para cristalização do PCL. A Fig. 11b corresponde a blenda P(3-HB)/PCL (80/20) é bem semelhante a blenda anterior, onde se observa separação de fases e a presença de esferulitos dos dois polímeros. A Fig. 11e, que corresponde a blenda P(3-HB)/PCL (20/80) também mostra separação de fases. Observa-se que a parte mais escura corresponde aos esferulitos da PCL, enquanto a parte mais clara é relativa aos esferulitos de $\mathrm{P}(3-\mathrm{HB})$.

\section{Conclusões}

A técnica de MDSC informa a presença de duas temperaturas de transição vítrea, que não se alteram, o que indica que as blendas são imiscíveis. Conforme observado por microscopia eletrônica de varredura, a morfologia variou de forma significativa em função da composição das blendas. As composições 20/80 e 80/20 apresentaram morfologia similar à do polímero presente em maior concentração. Com $50 \%$ de PCL, a morfologia da blenda mostra a presença microesferas. A microscopia óptica com luz polarizada mostrou a presença de esferulitos dos dois polímeros, que também se separam em duas fases distintas, concordando com os resultados da análise térmica.

\section{Agradecimentos}

Gostaríamos de agradecer ao Prof. Hugo Alejandro Gallardo Olmedo (UFSC) pelo uso do Microscópio Óptico, ao $\mathrm{CNPq}$ pela bolsa de recém doutor da Pesquisadora A.P. 
T. Pezzin, ao FUNCITEC pelo financiamento do projeto e à UNIVILLE pelo financiamento do projeto e pelas bolsas de iniciação científica dos alunos Nilton Vogelsanger e Michele Formolo.

\section{Referências Bibliográficas}

1. Otake, Y.; Kobayashi, T.; Asabe, H.; Murakami, N.; ONO, K. J. Appl. Polym. Sci, v. 56, p. 1789-1796, 1995.

2. Abe, H.; Doy, Y. International Journal of Biological Macromolecules, v. 25, p. 185-192, 1999.

3. Darwis, D.; Minoto, H.; Enjoji, T.; Yoxhii, F.; Makuuchi, K. Polymer Degradation and Stability, v. 62, p. 259-265, 1998.

4. Satoh, H.; Yoshie, N.; Yoshio, I. Polymer, v. 35, n. 2, p. 286-289, 1994.

5. Yoshie, N.; Nakasato, K.; Fujiwara, M.; Kasuya, K.; Abe, H.; Doy, Y.I. Polymer, v. 41, p. 3227-3234, 2000.

6. Zhang, L.L.; Goh, S.H.; Lee, S.Y.; Hee, G.R. Polymer, v. 41. p. 1429-1439, 2000.

7. Marangoni, C.; Furigo Jr, A.; Aragão, G.M.F. Biotechnology Letters, v. 22, p. 1635-1638, 2000.
8. Braunegg, G.; Lefbvre, G.; Genser, K.F. Journal of Biotechnology, v. 65, p. 127-61, 1998.

9. Kumagai, Y.; Doi, Y. Polymer Degradation and Stability, v. 37, p. 253-256, 1992.

10. Pezzin, A.P.T.; Zavaglia, C.A.C.; Duek, E.A.R. Polímeros: Ciência e Tecnologia, v. 12, n. 4, p. 285-294, 2003.

11. Pezzin, A.P.T.; Duek, E.A.R. Polymer Degradation and Stability, in press, 2003.

12. Gassner, F.; Owen, A.J. Polymer Report, v. 35, p. 2233-2236, 1994.

13. Savenkova, L.; Gercberga, Z.; Bibers, I.; Kalnin, M. Process Biochemistry, v. 36, p.445-450, 2000.

14. Ikejima, T.; Inoue, Y. Carbohydrate Polymers, v. 41, p. 351-356, 2000.

15. Xu, J.; Guo, B.H.; Yang, R.; Wu, Q.; Chen, G.Q.; Zhang, Z.M. Polymer, v. 43, p. 6893-6899, 2002.

15. Quental, A.C.; Felisberti, M.I. Anais do $6^{\circ}$ Congresso Brasileiro de Polímeros, p. 1260-1263, 2001.

16. Tsuji, H.; Ikada, Y. Journal of Applied Science, v. 60, p. 2367-2375, 1996. 